\title{
Optical methods of silicon nanoparticle diagnostics for applications in biomedicine
}

\author{
Alida F. Alykova ${ }^{1 *}$, Valery G. Yakunin ${ }^{2}$, Victor Yu. Timoshenko ${ }^{1,2,3}$, and Irina N. Zavestovskaya ${ }^{1,3}$ \\ ${ }^{1}$ National Research Nuclear University MEPhl (Moscow Engineering Physics Institute), 31 Kashirskoe shosse, \\ Moscow 115409, Russian Federation \\ ${ }^{2}$ Lomonosov Moscow State University, GSP-1 Leninskie Gory, Moscow 119991, Russian Federation \\ ${ }^{3}$ P. N. Lebedev Physical Institute of the Russian Academy of Sciences, 53 Leninskiy Prospekt, Moscow 199991, Russian \\ Federation
}

*e-mail: AFAlykova@mephi.ru

\begin{abstract}
The method of Raman scattering spectroscopy was used to study various silicon nanostructures (nanowires, mesoporous nanoparticles, crystalline and laser-ablated nanoparticles) dispersed in aqueous medium. The obtained results indicate different dissolution rate for silicon nanoparticles of different sizes and morphology in water that can be used for their potential biomedical applications. (C) 2019 Journal of Biomedical Photonics \& Engineering.
\end{abstract}

Keywords: silicon nanocrystals; nanowires; porous silicon; nanocrystalline silicon; laser ablated nanoparticles; Raman scattering; biodegradation.

Paper \#3325 received 24 Apr 2019; revised manuscript received 4 Jun 2019; accepted for publication 4 Jun 2019; published online 29 Jun 2019. doi: 10.18287/JBPE19.05.020304.

\section{Introduction}

In recent years, various types of nanostructured forms of crystalline silicon ( $\mathrm{Si}$ ) have been intensively studied for application in technology and biomedicine, including for the diagnosis and therapy of various diseases [1, 2]. Porous $\mathrm{Si}$ [3, 4-7], silicon nanowires [8], and nanoparticles (NPs) obtained from them [9], as well as laser-ablated Si NPs [2, 10] and nanoparticles obtained from porous c-Si [11], have a great potential of unique physical and chemical properties for application both in tumor therapy $[1,2,4,9]$ and as contrast agents for bioimaging $[8,10,11]$.

At present, several methods for obtaining powders and stable aqueous suspensions of Si NPs are known $[1-6,9,10]$. Photoluminescence (PL) of laser-ablated Si NPs, which is explained by radiative recombination of excitons excited in silicon nanocrystals with dimensions of 3-5 nm, was successfully applied in the study of the NP penetration into living cells [10]. It was found that Si NPs can be effectively accumulated in cancer cells and therefore they be used for diagnostics and therapy of cancer [2]. It was shown that the processes of accumulation and excretion of Si NPs in biological systems can be evaluated by the Raman spectroscopy [9].

In our work, we use the Raman scattering spectroscopy to study an effect of water exposure on the structural properties of crystalline $\mathrm{Si}$ nanowires (SiNWs), mesoporous silicon (meso-PSi), silicon nanocrystals (nc-Si) obtained by plasma-chemical synthesis or by the method of femtosecond laser ablation of c-Si targets in water (abl-Si).

\section{Experimental technique}

Samples of SiNWs were formed by metal-stimulated chemical etching (MSCE) of single-crystal silicon wafers $(\mathrm{c}-\mathrm{Si})$ of p-type conductivity with resistivity of $1-10 \mathrm{Om} \times \mathrm{cm}$ and surface orientation (100) by using the standard two step approach (see e.g. Ref. [8]).

Meso-PSi samples were formed using the standard method of electrochemical etching plates c-Si p-type conductivity with surface orientation (100) and resistivity of $1-5 \mathrm{mOm} \times \mathrm{cm}$ mixture of hydrofluoric acid and ethanol $\left(\mathrm{HF}(50 \%): \mathrm{C}_{2} \mathrm{H}_{5} \mathrm{OH}=1: 1\right)$ at a current density $60 \mathrm{~mA} / \mathrm{cm}^{2}$ for 1 hour.

After that, meso-PSi films were separated from the silicon substrate by a short-term increase in the current density up to $600 \mathrm{~mA} / \mathrm{cm}^{2}$. Aqueous suspensions of nanoparticles were obtained as a result of mechanical grinding of meso-PSi films in the planetary ball mill FRITSCH "Pulverisette 7" for 9 minutes at a rotation speed of $800 \mathrm{rpm}$ and a diameter of $5 \mathrm{~mm}$ zirconium oxide balls used (see, for example, review [3]). Powders of nc-Si were obtained by the method of plasma- 
chemical synthesis with the use of pure (99.9\%) microcrystalline $\mathrm{Si}$ as the feedstock (see, for example, Ref. [1]).

The samples of SiNWs were placed in a $4 \mathrm{~cm}^{3}$ cuvette filled with deionized water or saline $(0.9 \% \mathrm{NaCl}$ in $\mathrm{H}_{2} \mathrm{O}$ ) and held for a number of days. As a starting material, a sample with lateral sample sizes of about $1 \times 1 \mathrm{~cm}$ and a nanowire length of about $10 \mu \mathrm{m}$ was used. To study the dissolution of PF, aqueous suspensions of meso-PSi, nc-Si and abl-Si with a concentration of about $1 \mathrm{~g} / 1$ in a volume of $10 \mathrm{ml}$ were placed in dialysis bags with pore sizes of 6-10 kDa and kept from 1 to 24 hours in a large volume (5 liters) of deionized water at room temperature. Then at intervals $1,2,3,6,12,24$ hours some part of the suspension was collected, were planted on the stainless steel substrate, air-dried for 10-15 min and then examined by using the Raman scattering. All experiments were conducted at room temperature.

The Raman spectra of the obtained samples were taken on the DFS-52 spectrometer, which is located in the laboratory of the Department of low temperatures and superconductivity of MSU, in 90-degree scattering geometry. The receiver served working in the photon counting mode a cooled photomultiplier tube PMT-79. As a source of exciting radiation, an argon laser with wavelengths of 488 and $514.5 \mathrm{~nm}$ and a maximum intensity of not more than $100 \mathrm{~W} / \mathrm{cm}^{2}$ was used. The HORIBA Jobin Yvon LabRAM HR Visible microRaman spectrometer was used to measure Raman scattering spectra with excitation at a wavelength of $632.8 \mathrm{~nm}$. The Raman spectra were also measured at the "Horiba Jobin Yvon" MicroRaman LabRAM HR Visible facility when excited by $\mathrm{HeNe}$ laser radiation with a wavelength of $632.8 \mathrm{~nm}$ or an argon laser with a wavelength of $488 \mathrm{~nm}$. The signal was recorded in the backscattering geometry at normal incidence at room temperature in the air.

\section{Results and discussion}

Raman spectra of silicon nanowires with average crosssectional dimensions of $20-100 \mathrm{~nm}$ before and after exposure to water or normal saline for 2 weeks are shown in Fig. 1. In the spectrum, there is a line at $520.5 \mathrm{~cm}^{-1}$ with width about $4 \mathrm{~cm}^{-1}$, corresponding to the scattering on optical phonons of the c-Si crystal lattice. The high intensity of the line in comparison with single crystals of $\mathrm{c}-\mathrm{Si}$ indicates the penetration of exciting radiation into the SiNWs layer followed by multiple scattering. The forms of the Raman scattering line in both c-Si and SiNWs samples are well described by the Lorentz function:

$$
I_{c}(\omega)=\frac{C}{\left(\omega-\omega_{c}\right)^{2}+\left(\frac{1}{2} \Delta \omega_{c}\right)^{2}},
$$

where $\omega_{c}=520.5 \mathrm{~cm}^{-1}$ is the Raman phonon frequency in c-Si $\mathrm{s}, \Delta \omega_{c}=3 \mathrm{~cm}^{-1}$ is the width of Raman line of c-Si at room temperature, $\mathrm{C}$ is a constant.

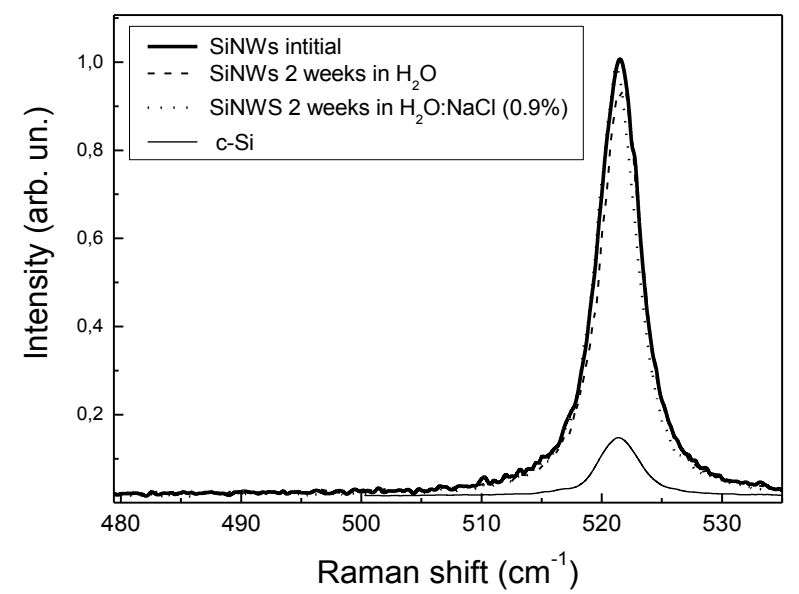

Fig. 1 Raman spectra of SiNWs before (thick solid line) and after storage in water (dotted line) and saline (punch line) for 2 weeks, as well as, for comparison, for c-Si substrate (thin solid line).

Obviously that the silicon nanowires in water and physiological (normal) saline solution do not exhibit a significant change of signal of Raman scattering which indicates the stability of the nanowires to dissolution. This stability is explained by the significant size of the cross section, as well as a sufficiently high quality oxide coating that occurs at the final stage of production.

When the samples are in water for one day meso-PSi silicon nanoparticles with the size of nanocrystals of about $10 \mathrm{~nm}$ demonstrate a multiple decrease in the intensity of Raman scattering, a shift in the band and an increase in the contribution of the amorphous phase of silicon, which indicates an effective dissolution (Fig. 2).

In accordance with Raman spectroscopy for mesoPSi we use the model proposed in Ref. [13]. According to this model limitations of the phonons to the nc-Si, we can use the following equation to form the line of Raman scattering [12, 13]:

$$
I_{N C}(\omega)=\int_{0}^{1} \frac{B \exp \left(-\frac{1}{4} q^{2} L^{2}\right) q^{2}}{\left(\omega-\omega_{c}(q)\right)^{2}+\left(\frac{1}{2} \Delta \omega_{c}\right)^{2}} d q,
$$

where $\omega_{c}=520.5 \mathrm{~cm}^{-1}$ frequency phonons, the parameter $L=d / a_{0}, d$ is the diameter, $a_{0}=0.543 \mathrm{~nm}$ is the lattice constant of c-Si, $q$ - the wave vector of the Phonon, is expressed in units $\left(2 \pi / a_{0}\right.$, and $\left.\omega_{c}(q)=\omega_{c}\left(1-0.18 q^{2}\right)\right)$ the ratio of the dispersion of the phonon in c-Si [13], B is a constant.

Eq. (2) describes the confinement effect (phonon restriction effect) in spherical Si nanocrystals with sizes 1-10 nm [12], while for Raman scattering with large nanoparticles the expression (1) can be used. 


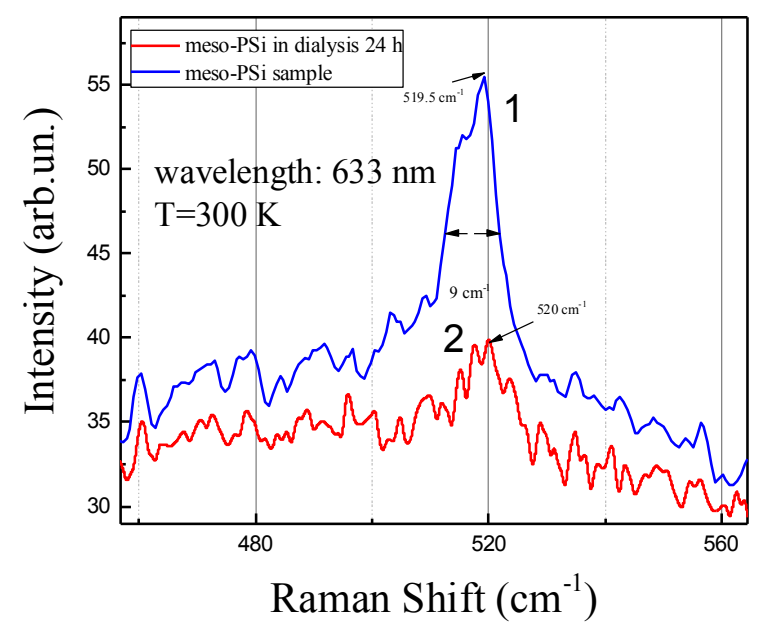

Fig. 2 Raman spectra for the initial meso-PSi (1) sample and after dialysis in water for $24 \mathrm{~h} \mathrm{(2).}$

The Raman spectrum of amorphous silicon (a-Si) is described by the following expression [13]:

$$
I_{A}(\omega)=A \times \exp \left(-\frac{\left(\omega-\omega_{a}\right)^{2}}{2 \delta^{2}}\right),
$$

where $A$ is a constant, $\omega_{a}=480 \mathrm{~cm}^{-1}$ and $\delta=\Gamma(2 \sqrt{2 \ln 2})^{-1} \quad \Gamma=70 \mathrm{~cm}^{-1}$ are the peak position width, respectively.

Assuming that the Raman scattering signal of the sample under study consists of inclusions of all fractions (phases) the forma can be presented as follows:

$$
I_{\text {Sum }}(\omega)=I_{c}(\omega)+I_{N C}(\omega)+I_{A}(\omega)
$$

The volume fraction of the nc-Si phase can be calculated as the ratio of the corresponding integrated intensity to the total intensity of the Raman signal:

$$
f_{N C}=\int I_{N C}(\omega) d \omega / \int I_{\text {Sum }}(\omega) d \omega .
$$

The Raman spectra of meso-PSi analyzed by using Eqs. (1)-(4) show that the average size of $\mathrm{Si}$ nanocrystals is about $5 \mathrm{~nm}$ and it does not practically change after the storage of the sample in aqueous solution. However, the contribution of the nanocrystalline phase becomes smaller and the signal of a-Si fraction increased (see Fig. 3). The latter indicates an additional disordering of the crystal lattice of NP Si due to their partial dissolution in water in accordance with the findings of Ref. [9].

Investigation of Raman scattering from nonporous nc-Si with sizes from 5 to $100 \mathrm{~nm}$, obtained by plasma chemical (Fig. 3) and laser ablation methods (Fig. 4), indicates partial dissolution of nanoparticles, resulting in a decrease in their total number and average size of nanocrystals. In this case, abl-Si NPs exhibit significantly lower dissolution rate (Fig. 5), compared with NPs obtained by plasma-chemical method that can be explained by a higher concentration of structural defects in the latter.

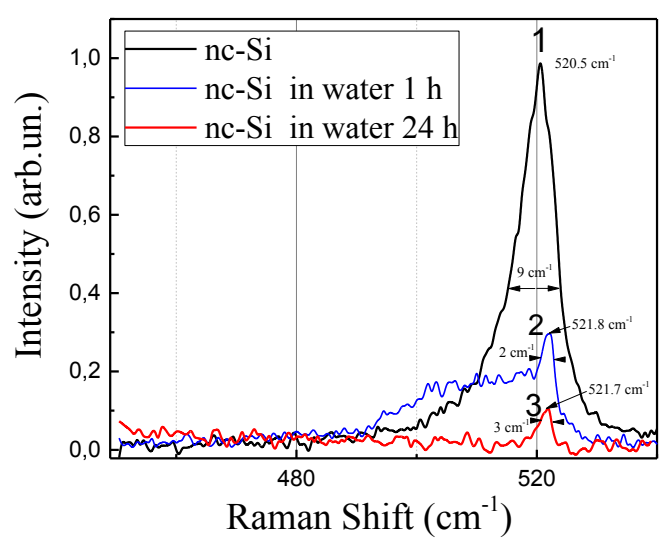

Fig. 3 Raman spectra of nc-Si samples before (1) and after exposure in water for 1 (2) and 24 (3) hours.

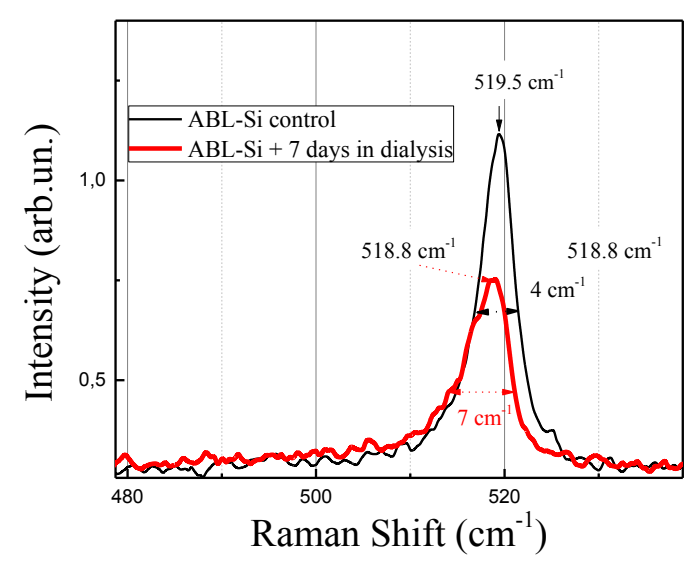

Fig. 4 Raman spectra of abl-Si samples before and after dialysis in water.

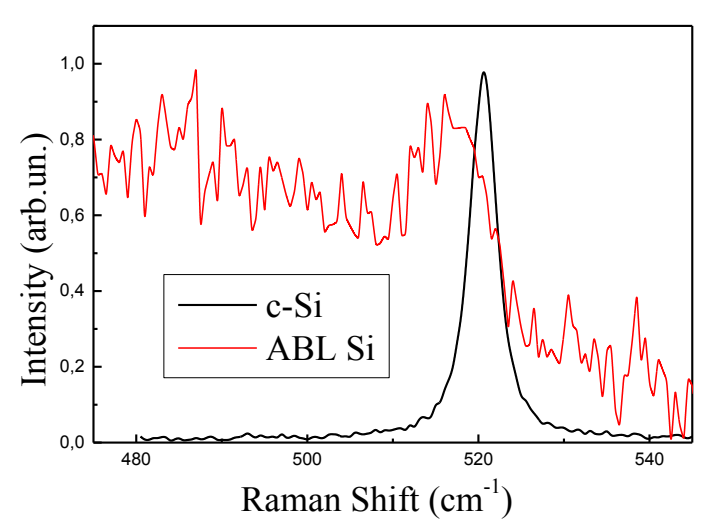

Fig. 5 Raman spectra of aqueous suspension of abl-Si source and c-Si substrate.

The obtained results indicate different dissolution rates of $\mathrm{Si}$ nanostructures prepared by wet chemical route and physical methods. Thus, SiNWs, due to their high stability in water, can be used as elements of biosensors. NPs of mesoporous silicon can be 
recommended for the purpose of express-diagnostics and therapy, while NPs produced by plasma-chemical synthesis and laser ablation of c-Si in water can be used as agents for short-time and prolonged simultaneous diagnosis and therapy (theranostics) of cancer diseases.

\section{Disclosures}

All authors declare that there is no conflict of interests in this paper.

\section{Acknowledgments}

The authors thank Prof. A. A. Ishchenko (MIREA Russian University of technology) for providing the samples obtained by plasma-chemical method, Dr. A.V. Pavlikov for the assistance in selected measurements on the equipment of the Faculty of Physics of Moscow State Lomonosov University.

The work was supported by the state tasks of the Ministry of science and higher education of the Russian Federation (projects 16.2969.2017/4.6 and 16.7917.2017/8.9).

\section{References}

1. A. A. Ischenko, G. V. Fetisov, and L. A. Aslanov (eds.), Nanosilicon: Properties, Synthesis, Applications, Methods of Analysis and Control, CRC Press, Taylor\& Francis Group, Boca Raton, London, New York (2015).

2. A. V. Kabashin, V. Y. Timoshenko, "What theranostic applications could ultrapure laser-synthesized Si nanoparticles have in cancer?” Nanomedicine 11(17), 2247-2250 (2016).

3. L. A. Osminkina, V. Y. Timoshenko, "Porous Silicon as a Sensitizer for Biomedical Applications: Mini-review," Open Material Sciences 3(1), 39-48 (2016).

4. L. A. Osminkina, A. A. Kudryavtsev, S. V. Zinoviev, A. P. Sviridov, Y. V. Kargina, K. P. Tamarov, V. N. Nikiforov, A. V. Ivanov, A. N. Vasiliev, and V. Y. Timoshenko, "Silicon nanoparticles as amplifiers of ultrasonic action for sonodynamic therapy," Bulletin of experimental biology and medicine 161(2), 296-299 (2016).

5. A. P. Sviridov, L. A. Osminkina, A. L. Nikolaev, A. A. Kudryavtsev, A. N. Vasiliev, and V. Y. Timoshenko, "Lowering of the cavitation threshold in aqueous suspensions of porous silicon nanoparticles for sonodynamic therapy applications," Applied Physics Letters 107(12), 123107 (2015).

6. M. B. Gongalsky, Y. V. Kargina, L. A. Osminkina, A. M. Perepukhov, M. V. Gulyaev, A. N. Vasiliev, Y. A. Pirogov, A. V. Maximychev, and V. Y. Timoshenko, "Porous silicon nanoparticles as biocompatible contrast agents for magnetic resonance imaging," Applied Physics Letters 107(23), 233702 (2015).

7. L. A. Osminkina, A. L. Nikolaev, A. P. Sviridov, N. V. Andronova, K. P. Tamarov, M. B. Gongalsky, A. A. Kudryavtsev, H. M. Treshalina, and V. Y. Timoshenko, "Porous silicon nanoparticles as efficient sensitizers for sonodynamic therapy of cancer," Microporous \& Mesoporous Materials 210, 169-175 (2015).

8. S. P. Rodichkina, L. A. Osminkina, M. Isaiev, A. V. Pavlikov, A. V. Zoteev, V. A. Georgobiani, K. A. Gonchar, A. N. Vasiliev, and V. Y. Timoshenko, "Raman diagnostics of photoinduced heating of silicon nanowires prepared by metal-assisted chemical etching," Applied Physics B 121(3), 337-344 (2015).

9. E. Tolstik, L. A. Osminkina, C. Matthäus, M. Burkhardt, K. E. Tsurikov, U. A. Natashina, V. Y. Timoshenko, R. Heintzmann, J. Popp, and V. Sivakov, "Studies of silicon nanoparticles uptake and biodegradation in cancer cells by Raman spectroscopy,” Nanomedicine: Nanotechnology, Biology \& Medicine 12(7), 1931-1940 (2016).

10. M. B. Gongalsky, L. A. Osminkina, A. Pereira, A. A. Manankov, A. A. Fedorenko, A. N. Vasiliev, V. V.Soloviev, A. A. Kudryavtsev, M. Sentis, A. V. Kabashin, and V. Y. Timoshenko, "Laser-synthesized oxide-passivated bright Si quantum dots for bioimaging," Scientific Reports 6(1), 24732 (2016).

11. A. Kharin, O. Syshchyk, A. Geloen, S. Alekseev, A. Rogov, V. Lysenko, and V. Timoshenko, "Carbon fluoroxide nanoparticles as fluorescent labels and sonosensitizers for theranostic applications," Science and Technology of Advanced Materials 16(4), 044601 (2015).

12. H. Richter, Z. P. Wang, and L. Ley, "The one phonon Raman spectrum in microcrystalline silicon," Solid State Communications 39(5), 625-629 (1981).

13. N. E. Maslova, A. A. Antonovsky, D. M. Zhigunov, V. Y. Timoshenko, V. N. Glebov, and V. N. Seminogov, "Raman studies of silicon eanocrystals embedded in silicon suboxide layers," Semiconductors 44(8), 1040-1043 (2010).

14. A. F. Alykova, I. N. Zavestovskaya, V. G. Yakunin, and V. Y. Timoshenko, "Raman diagnostics of silicon nanocrystals dissolution in aqueous medium," Journal of Physics: Conference Series 945, 012002 (2018). 